\title{
Un nuevo sistema de diseño de embuticiones en láminas de acero para maximizar resistencia de losas compuestas
}

\section{A new system for designing embossments in steel sheets to increase composite slabs shear resistance.}

\author{
Melchor López Ávila*1, Rafael Larrúa Quevedo**, Carlos Recarey Morfa*** \\ * U niversidad Central "Marta Abreu” de lasVillas, melchor@uclv.edu.cu \\ ** Universidad de Camagüey, Camagüey, CU BA \\ *** Centro Internacional de M étodos Computacionales y Numéricos en la Ingeniería. Universidad Central de Las Villas, \\ Santa Clara, CUBA
}

Resumen

\begin{abstract}
Se presenta un resumen de algunos de los principales antecedentes históricos en el análisis de las losas compuestas con láminas metálicas colaborantes sometidas a flexión, así como de los principales métodos de diseño, instrumentación y ensayo aceptados por las distintas normas internacionales. Se analizan cada uno de los parámetros fundamentales que influencian el comportamiento estructural de las losas compuestas, y a partir del estudio numérico de estos se propone un método de optimización del sistema de embuticiones, implementado en hojas de cálculo, con el cual es posible estimar una resistencia a esfuerzos cortantes para las láminas de perfilado abierto y entregando los resultados de los parámetros más importantes, y que empleándolos en forma comparativa es posible determinar el sistema de embuticiones óptimo a incluir en una lámina de perfilado abierto. Aplicando el método propuesto obtuve un sistema de embuticiones tecnológicamente posible y con un coste mínimo de inversiones para una línea industrial de láminas de perfilado abierto.
\end{abstract}

Palabras Clave: Compuestas, losas, embuticiones, lámina, diseño

Abstract

A summary is presented with the main historical background in the analysis of the composite slabs with steel sheets subjected to flexion, as well as of the main methods of design, instrumentation and tests accepted by different International Standards. They fundamental parameters that influence the structural behaviour of the composite slabs are analyzed, and with a numeric study an analytical optimization method of the embossments system, is proposed and implemented in a spread sheet is possible to estimate the shear resistance of open web sheets and by comparing them, is possible to determine the better embossments system to include in a open web sheet with such method. Applying the proposed method a technologically possible embossments system can be obtained with a minimum cost for an industrial line of open web sheets.

Keyw ords: Composite, slabs, embossments, sheet, design

\section{Introducción}

Los métodos de diseño para losas compuestas con láminas metálicas colaborantes consisten regularmente en ensayos a escala natural, y posteriormente empleando métodos semiempíricos se determinan coeficientes que caracterizan el diseño de la losa compuesta con el tipo de lámina metálica o steel deck específico con que se ejecutó la prueba experimental.

Para las empresas productoras de láminas, el desarrollar un nuevo prototipo o producto resulta entonces extremadamente costoso, puesto que se tendría que

\footnotetext{
1 Autor de correspondencia / Corresponding autor:

Edificio 21. Apartamento C1. Reparto Aguilera. Las Tunas, CP 75300. Cuba. Teléfono: 5303134 8012, E-mail: melchor@uclv.edu.cu
}

recurrir primeramente a la producción de la lámina tipo con el sistema de embuticiones que esta tendría, para con este producto experimental realizar los ensayos a escala natural y verificar entonces si resulta competitivo o no.

Evidentemente, estos métodos resultan bastante caros e inexactos en cuanto a determinar el sistema de embuticiones óptimo a practicar en una lámina metálica lisa que se pretenda transformar en lámina colaborante o steel deck, lo cual dificulta la creación de nuevos productos con la máxima eficiencia estructural posible.

En el presente trabajo proponemos un método 
completamente analítico con el cual es posible determinar el sistema de embuticiones óptimo a practicar en una lámina metálica de perfilado abierto para que ésta pueda utilizarse con fines colaborantes en las losas compuestas, así como su aplicación en la creación de un nuevo producto a partir de la tecnología existente en Cuba para producir láminas grecadas trapezoidales.

\section{Estado del arte}

Una losa compuesta es aquella en la que se utilizan láminas de acero como encofrado colaborante capaces de soportar el hormigón vertido, la armadura metálica y las cargas de ejecución. Posteriormente las láminas de acero se combinan estructuralmente con el hormigón endurecido y actúan como armadura a tracción en el forjado acabado, comportándose como un elemento estructural mixto hormigón- acero (Figura 1).

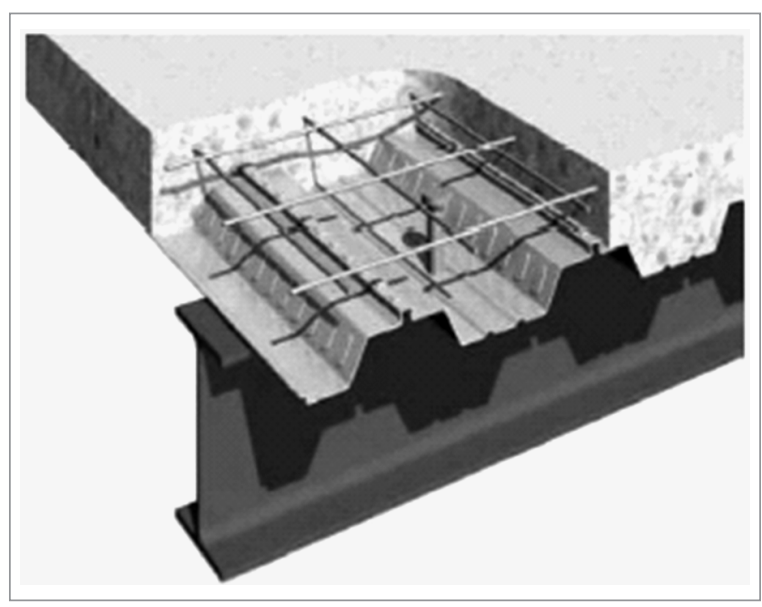

Figura 1. Losa compuesta

La utilización de láminas metálicas en la construcción de entrepisos se remonta a los años 20. En dichas construcciones la lámina metálica regularmente constituía el principal componente estructural. La adición de una cubierta de hormigón trajo consigo no sólo aumentar la resistencia estructural, sino que también sirvió a los propósitos de protección contra el fuego, como una manera de nivelar la superficie del piso y como una forma de distribuir las cargas más uniformemente. U na filosofía contrastante en el empleo de las láminas metálicas era que éstas se empleaban sólo en función de encofrados perdidos y que la losa de hormigón armado sobre ellas fundida era quien supuestamente aportaba toda la capacidad estructural. El concepto de emplear losas compuestas con las láminas metálicas con funciones colaborantes no surge sino hasta los años 50 (Chen, 2003). Las losas compuestas formadas por láminas de acero como encofrado colaborante constituyen soluciones muy eficientes. Son numerosas las ventajas que ofrece esta tipología estructural. Entre ellas podemos resaltar:

- La sección compuesta en entrepisos aprovecha al unísono la resistencia a compresión del hormigón y la resistencia a tracción del acero, con lo cual se logra que para las mismas cargas y luces se requieran menores secciones de perfiles estructurales.

- Con el empleo de las estructuras compuestas se logra mayor avance físico en la ejecución de las obras, permitiendo aprovechar además la lámina metálica de encofrado como posterior refuerzo de acero permanentemente en la losa de hormigón.

- Debido a que la lámina metálica colaborante asume la función de encofrado, se logra un ahorro casi total de madera por este concepto, lo cual repercute como impacto ambiental.

- Se requiere menor cantidad de operarios calificados, lo que implica un ahorro económico sustancial en cuanto a fuerza de trabajo.

- La lámina metálica colaborante, en función adicional de encofrado y plataforma de trabajo, ofrece grandes facilidades para la construcción y montaje de instalaciones eléctricas e hidráulicas.

- La sección compuesta incrementa la rigidez y disminuye las flexiones con respecto a los elementos individuales.

- Debido al trabajo conjunto hormigón- lámina se permite reducir los espesores de hormigón en las losas de entrepiso, disminuyendo con esto las cargas debidas al peso propio y por consiguiente, ahorro en costos de estructura, mampostería, cimentación, etc.

- Como resultado del diseño compuesto, el peso de las vigas metálicas puede reducirse desde un 5 hasta un $30 \%$, optimizando los diferentes materiales (Hernández, 2003).

Las losas compuestas son sistemas en los cuales la lámina metálica, debido a la interacción mecánica que manifiesta con el hormigón endurecido, actúa como refuerzo positivo a flexión.

En las losas compuestas se presentan tres modos de fallo principales:

1- Fallo a flexión

2- Fallo por cortante vertical

3- Fallo por cortante longitudinal 
El fallo por cortante longitudinal es el más probable de ocurrir y se caracteriza por la formación de grietas diagonales en la masa de hormigón (Figura 2), próximas a los puntos de aplicación de la carga y acompañados por deslizamientos en los puntos extremos. La distancia desde estas fisuras hasta los apoyos se denomina "luz de cortante" $(L ')$.

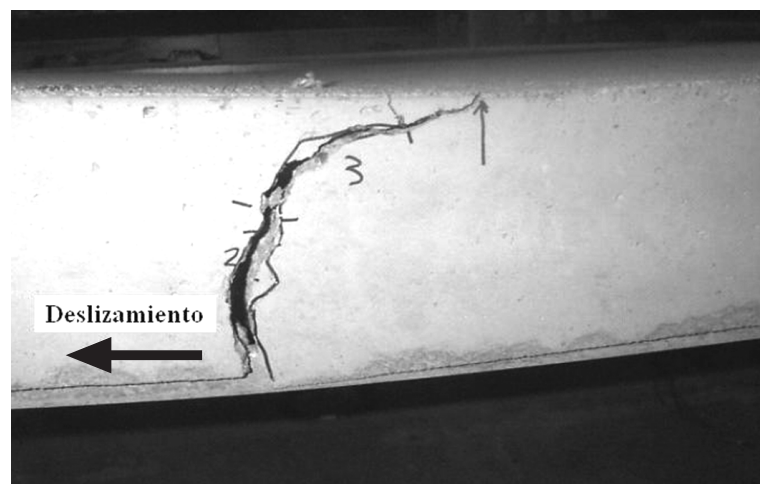

Figura 2. Fallo por cortante longitudinal

El fallo del enlace a cortante, con incremento paulatino de la carga aplicada, ocurrirá con la siguiente secuencia (Seleim y Schuster 1985):

1. Los dispositivos de transferencia de cortante comienzan a recibir esfuerzos cada vez mayores (Figura 3).

2. Aparecen las primeras grietas en la sección crítica, incrementándose la diferencia de tensiones entre el hormigón y la lámina. La lámina empieza a separarse de la losa perdiendo la efectividad del trabajo conjunto. Las fisuras se hacen cada vez mayores.

3. Fallan completamente los dispositivos de transferencia de cortante, traduciéndose en deslizamientos.

4. El grado de fisuración se hace inaceptable, y la lámina metálica y la losa de hormigón se separan completamente, empezando a trabajar como elementos independientes, perdiéndose el concepto de trabajo compuesto.

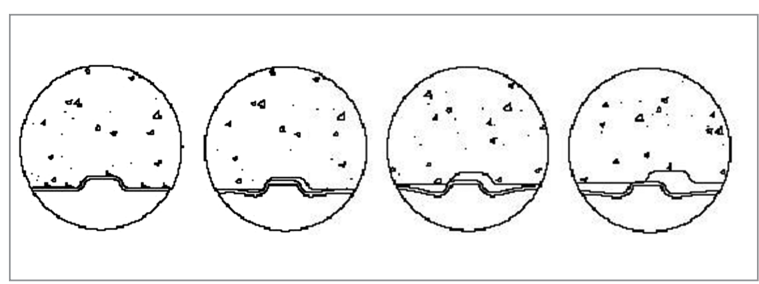

Figura 3. Secuencia de fallo

El ensayo más incuestionable para determinar la capacidad resistente de las losas compuestas es el de flexión a escala natural, desarrollado por la AISI en 1967 (AISC, 2007; ASTM-E8-00b, 2001), y en base a este procedimiento se han desarrollado tres ecuaciones principales para calcular el cortante de diseño.

$\frac{V_{u} s}{b d}=m \frac{d \sqrt{f^{\prime}}}{L^{\prime}}+k \rho \quad($ Schuster, 1970)

$\frac{V_{u} s}{b d}=m \frac{\rho d}{L^{\prime}}+k{\sqrt{f^{\prime}}}_{c} \quad($ Porter y Ekberg 1975; 1976)

$\frac{V_{u}}{b d}=k_{1} \frac{t}{L^{\prime}}+k_{2} \frac{1}{L^{\prime}}+k_{3} t+k_{4} \quad$ (Seleim, 1979)

donde:

$V_{u=}$ Resistencia última de enlace transversal a cortante (por unidad de ancho)

$\mathrm{b}=$ Ancho unitario de la losa

$\mathrm{d}=$ Profundidad efectiva de la losa

$\mathrm{s}=$ Espaciamiento de los dispositivos de transferencia de cortante

$\mathrm{m}, \mathrm{k}=$ Parámetros determinados experimentalmente $\rho=$ Proporción de refuerzo de acero,

$L^{\prime}=$ Longitud de la luz de cortante, $\frac{A_{s}}{b d}$

$\mathrm{f}^{\prime}{ }_{\mathrm{c}}=$ Resistencia a compresión del hormigón

La luz de cortante, $L$ ', es la longitud crítica para la cual ocurre el fallo por cortante Iongitudinal. $L^{\prime}$ usualmente se toma como la cuarta parte de la luz total para el caso de una losa cargada uniformemente, sin embargo, algunos autores argumentan que esta debe ser la tercera parte de la luz (Tenhovouri y Leskela, 1998; Veljkovic, 2000). Para el caso de cargas concentradas en dos puntos, la luz de cortante será la distancia medida desde el apoyo hasta el punto de aplicación de la carga (Porter y Ekberg 1976; Porter y Ekberg, 1975).

El método asumido por la norma cubana NC 082:2004 coincide con el segundo de los explicados anteriormente, el de Porter y Ekberg (NC-082, 2004).

En 1985 Seleim y Schuster evaluaron esas tres ecuaciones a partir del ensayo a 196 especímenes y concluyeron que la ecuación de Seleim (CSSBI-S3, 2003) es más precisa que las otras dos.

Estas expresiones están dadas para losas de luces simples. Las probetas de losas continuas ofrecen capacidades de carga superiores entre un 10 y un $15 \%$ que sus homólogas de luces simples. (Luttrell, 1987; Abdullah, 2004).

La resistencia a cortante la constituyen tres 
componentes: la adherencia química, la fricción, y la interacción mecánica. Los efectos de fricción resultan mayores sobre los apoyos debido a que allí también son mayores las fuerzas normales, no obstante la fricción actúa a todo lo largo de la luz (Schuurman y Stark 1996; Veljkovic, 1994). Cuando no conocemos con precisión el valor del coeficiente de fricción, este puede ser asumido entre 0.5 y 0.6 (Bode y Dauwel, 1999; Kitoh y Sonoda 1996 ). La adherencia química resulta extremadamente baja y difícil de predecir, empezando porque depende del proceso de curado del hormigón y de las condiciones superficiales de la lámina. Por su parte, la interacción mecánica ofrece resistencia al deslizamiento debido a los obstáculos en su curso, tales como las embuticiones de la lámina, las mallas de refuerzo de acero soldadas transversalmente a la chapa (práctica no habitual debido al encarecimiento de los costos), los huecos practicados en la lámina metálica y/o los conectores de cortante.

El ensayo de probetas a escala natural resulta muy costoso tanto en gasto de materiales y utilización de mano de obra como en consumo de tiempo, y al parecer esas fueron las causas que motivaron a los investigadores a desarrollar modelos de ensayos en escala reducida. Estos ensayos se enfocan en determinar la magnitud del enlace a cortante, que es quien usualmente determina el modo de fallo.

Los ensayos para determinar la resistencia a cortante resultan particularmente provechosos para comparar entre diferentes formas o dispositivos empleados para asumir la interacción mecánica durante el desarrollo de un nuevo modelo de producto. A partir de resultados experimentales, (Daniels, 1988) desarrolló un modelo de ensayo de tracción o pull out (Figura 4) así como ecuaciones para determinar las fuerzas de cortante en la interfase entre el hormigón y la lámina metálica, los cuales son bastante aceptados universalmente (Schumacher et al., 2000, Crisinel et al., 1999).

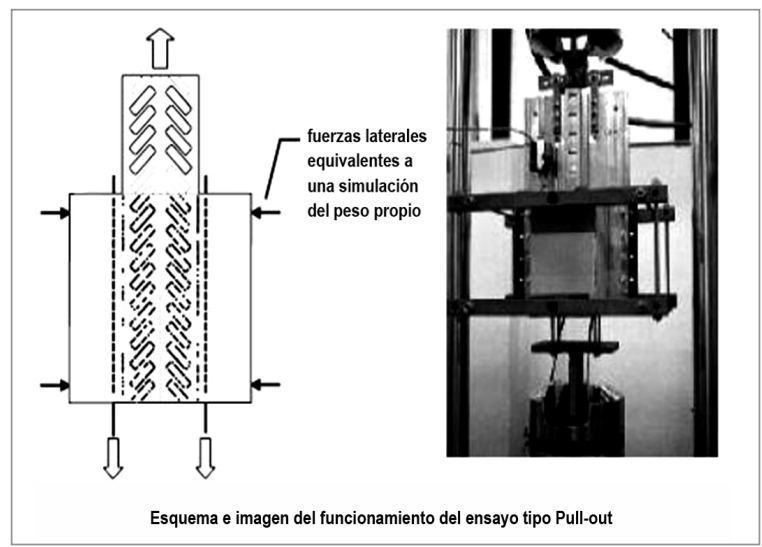

Figura 4. Ensayo pull -out de Daniels
SDI (Steel Deck Institute) compiló los resultados de varios proyectos de investigación y desarrolló un procedimiento de diseño de losas compuestas donde se demostró que $M_{\text {test }} / M_{n}$ se mantenía dentro del rango 1.01-1.31. Al procedimiento derivado se le llamó "de interacción parcial de cortante" (PSC) y con él se logran diseños más económicos que si se emplea el método mk (Bode y Dauwel 1999; Bode y Sauerborn, 1992; Eurocode-4, 2004).

En investigaciones más recientes se han comprobado algunas deficiencias del método PSC. Su aplicabilidad está limitada solamente a aquellas losas que presenten un fallo de tipo dúctil; esto obliga a que las luces cortas deban ser especialmente chequeadas debido a que las mismas tienden a presentar fallos frágiles. Para determinar la resistencia del enlace a cortante por este método, es necesario ejecutar ensayos a probetas en escala natural, lo cual, como ya se ha explicado anteriormente, es un proceso muy costoso tanto en materiales como en consumo de tiempo, espacio y mano de obra. El valor experimental de la resistencia a cortante depende del grado de interacción para el cual se desarrolla dicha resistencia, y la misma es altamente dependiente de las condiciones de carga de las pruebas experimentales (Edder y Crisinel, 2003; Veljkovic, 2000).

Varios han sido los investigadores que han empleado el método de elementos finitos para modelar el comportamiento de las losas compuestas. Veljkovic utilizó elementos tipo lámina (shell) en su modelo (Veljkovic y Johansson, 2001; Ferrer et al., 2005); por su parte, otros han preferido el método de fragmentación de vigas en secciones (Tenhovuori y Leskela, 1998), u otras formas de aprovechamiento de los métodos de particionamiento (Virdi, 2006; Ranzi et al., 2005; Abdullah, 2004).

Daniels, Crisinel, Laane y Edder (Laane y Edder, 2002; Crisinel et al., 1999; Daniels y Crisinel 1993) desarrollaron un método de análisis numérico que experimentalmente requiere sólo de ensayos en escala reducida para determinar la magnitud del enlace a cortante, y que es aplicable a losas de luces tanto simples como continuas. La principal ventaja radica en que este método no requiere de ensayos de flexión en escala natural. Con los resultados de los ensayos de tracción (pull- out) se calcula la resistencia de las embuticiones, y a partir de los ensayos de compresión (push- out) resulta posible estimar la resistencia que aportan los anclajes en los extremos. El comportamiento de cada sección transversal queda definido por dos parámetros, la fuerza axial y la curvatura. Este método además facilita que con un análisis 
no lineal por elementos finitos de la interacción parcial, sea posible estimar el comportamiento de la losa compuesta bajo las cargas actuantes. Los resultados que se obtienen por este método son muy similares a los que se logran aplicando el método de interacción parcial (PSC) o el método $\mathrm{m}-\mathrm{k}$.

Crisinel, Edder y Schumacher (Schumacher et al., 2000; Crisinel y Edder, 2004) desarrollaron un novedoso procedimiento al que llamaron Nuevo Método Simplificado (New Simplified Method). Su base está en crear un gráfico trilineal de la curvatura de momento en la sección crítica de la losa (Figura 5). Estas tres líneas representan una fase cada una. La primera, comportamiento elástico lineal: interacción completa entre la lámina y el hormigón, sin presencia de agrietamientos o deslizamientos. Segunda, comportamiento elástico o elasto- plástico: el hormigón empieza a agrietarse, pero en la probeta se conserva aún la completa interacción entre la lámina metálica y el bloque de hormigón. Y la tercera y última, comportamiento elastoplástico no lineal: el hormigón se ha fisurado, existen desplazamientos apreciables y se parcializa la interacción entre la lámina y el hormigón.

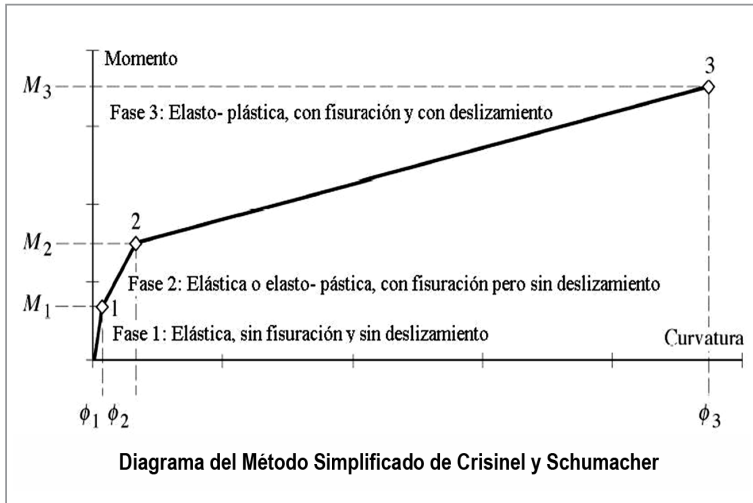

Figura 5. Diagrama de método simplificado de Crisinel

Este método brinda menos precisión para los especimenes de fallo frágil debido a la inconsistencia de la adherencia química, sin embargo, ha probado corresponder con los resultados experimentales con muy buena exactitud en los casos de probetas de losas compuestas con fallos en modo dúctil.

\section{Análisis de parámetros que influencian el comportamiento de las losas compuestas}

El interés actual de los estudios de losas compuestas se concentra en lograr láminas con sistemas de retención lo más eficientes como sea posible, de manera que permitan un mayor agarre con el bloque de hormigón y de esta forma dilaten el fallo por cortante longitudinal, que es el más probable de ocurrir. Evidentemente, optimizando las embuticiones, que pueden estar presentes en toda el área de la lámina, se perfeccionaría la interacción mecánica a todo lo largo y ancho de la losa compuesta. Los estudios experimentales desarrollados por diversos autores, entre los que sobresalen Ferrer y Marimón (Ferrer, 2006; Ferrer et al., 2005; Ferrer et al., 2007), Crisinel (Crisinel et al., 2006; Crisinel et al., 1999), Jolly y Zubair (Jolly y Zubair, 1987), Schumacher (Schumacher et al., 2000), M akelainen y Sun (Makelainen y Sun, 1999), Rossich (Rossich, 2005), Marimuthu (Marimuthu et al., 2006) Abdullah y Easterling (Abdullah y Easterling, 2007), han aportado las siguientes conclusiones respecto a los diseños de láminas:

1. Condiciones superficiales de la lámina: El acabado superficial de la lámina tiene una graninfluencia en la resistencia final al deslizamiento. La resistencia al deslizamiento en un ensayo Pull- out habiendo lubricado la superficie de la lámina ha resultado ser un $40 \%$ inferior que la resistencia de la lámina sin lubricar.

2. Ángulo de retención: Se define como el ángulo cerrado máximo que se produce en el perfil, teniendo en cuenta tanto el perfilado como el embutido (Figura 6). Es imprescindible disponer de un mínimo ángulo de retención, siendo especialmente crítico en los perfiles de ángulo abierto, puesto que el ángulo de retención depende entonces de la pendiente de embutición. El proceso de deslizamiento deforma la chapa pudiendo llegar a compensar rápidamente ángulos demasiado pequeños.

3. Ángulo de perfilado: Al afectar directamente al ángulo de retención en los perfilados de ángulo abierto, la resistencia al deslizamiento aumenta a medida que aumenta el ángulo de perfilado, puesto que se retarda la separación vertical convirtiéndose en reentrante en el límite. O bviamente las ventajas de un perfilado en ángulo abierto no son resistentes, sino de ahorro de material y de espacio de apilado para el transporte del producto.

4. Alternancia en el sentido de embutición: Se ha comprobado una mejora resistente notable al alternar el sentido de embutición consecutivamente en las embuticiones laterales de los perfiles en ángulo abierto, desde el hormigón hacia la chapa y desde la chapa hacia el hormigón. Con ello se consigue disponer de ángulo de retención en ambos extremos de las embuticiones alternadamente. Se disminuye también la dependencia resistente respecto al sentido 
de inclinación de las embuticiones y se aumenta el momento de inercia de la chapa respecto a la flexión transversal.

5. Inclinación de las embuticiones: No se observan motivos resistentes para inclinar las embuticiones laterales. Bien al contrario, resultan más eficaces las embuticiones sin inclinación y eliminan la alternancia de resistencias en nervios consecutivos, ya que el sentido de inclinación que favorece la separación vertical es menos resistente que el que tiende a juntar ambas partes.

6. Endiente de embutición: Este parámetro es el que mayor trascendencia tiene en la resistencia al deslizamiento. Afecta al ángulo de retención definido anteriormente en el caso de perfilados abiertos y relaciona las fuerzas normales a la chapa que provocan la flexión con la componente longitudinal que soporta el deslizamiento. Cuanto mayor es la pendiente de embutición, mayor es la componente longitudinal de fuerza necesaria para provocar el deslizamiento. Lógicamente, Ias limitaciones tecnológicas del proceso de embutido limitan el valor de este parámetro, así como la posible rotura del hormigón para valores de fuerza excesivos.

7. Profundidad de embutición: Es el parámetro causante de una mayor o menor flexión transversal en la lámina. Cuanto mayor sea la profundidad, mayor será la fuerza necesaria para deformar la lámina esa magnitud. A partir del punto en que la lámina se encuentra totalmente plastificada, los incrementos de profundidad no aumentan la resistencia. Como en el caso anterior, las limitaciones de profundidad de embutición vendrán determinadas por aspectos tecnológicos del proceso de embutición. Muy efectivo resulta incrementar la profundidad de las embuticiones, no obstante, deberá tenerse especial cuidado contra el desgarrado que pudiera ocurrir durante el proceso de producción.

8. Espesor de la lámina: El espesor de la lámina metálica es directamente proporcional a la resistencia a cortante Las simulaciones numéricas demuestran que en las láminas de perfilado abierto la dependencia de la resistencia al deslizamiento respecto al espesor no es lineal, sino que más bien se ajusta al cuadrado del espesor en la mayoría de casos. Esto resulta lógico si tenemos en cuenta que la caída de carga se debe a la plastificación de la lámina por flexión transversal. De aquí que la extrapolación lineal hacia espesores mayores esté siempre en el lado de la seguridad.

9. Proximidad de las embuticiones a las aristas del perfilado: La localización óptima de las embuticiones será en la mitad de la franja plana. Las embuticiones en las esquinas de las zonas planas o en las alas resultan muy difíciles de construir y no son del todo eficientes. Las embuticiones en las alas sometidas a tracción tienden a aplanarse bajo el efecto de las cargas, reduciendo así su efectividad. Por su parte, las embuticiones localizadas en las alas a compresión actúan como si fueran deformaciones iniciales, promoviendo así el efecto de pandeo

10. Ancho de las embuticiones: Cuanto mayor es el ancho de las embuticiones, mayor es el momento de inercia de la lámina con respecto a la flexión transversal, por tanto, mayores serán las fuerzas necesarias para deformarla. Además, afecta también a posibles roturas del hormigón, puesto que si la embutición es saliente, a mayor ancho, más resistente será la base de su negativo de hormigón. En cambio, en embuticiones entrantes debe tenerse cuidado en no debilitar el espacio entre embuticiones estrechándolo demasiado. La dimensión mínima de dicho espacio puede venir determinada por exigencias de diseño de la maquinaria de embutición y perfilado.

11. Distancia entre embuticiones (paso): Cuanto más próximas estén entre sí las embuticiones, mayor es el momento de inercia del conjunto de la lámina respecto a la flexión trasversal, y por tanto, más resistente, mostrando una fuerte dependencia lineal. El efecto es parecido a incrementar el ancho de las embuticiones, pero menos eficaz, ya que las embuticiones anchas alejan más cantidad de material del plano medio de la chapa que las embuticiones estrechas. Incrementar la frecuencia de las embuticiones a costa de reducir el tamaño de estas, no causa mejoramiento de la resistencia a cortante

12. Geometría de las embuticiones: Las discontinuidades en la forma de las embuticiones (tales como cruces) causa el efecto negativo de incrementar la flexibilidad de la lámina, promoviendo un más fácil deslizamiento del hormigón sobre esta.

Existen otros parámetros que también pudieran influenciar el comportamiento de las losas compuestas, como la resistencia del acero, pues se puede intuir que a mayor rigidez de la lámina metálica, mayores serán los esfuerzos necesarios para desalojarla de los negativos de embuticiones del hormigón, pero al parecer por razones tecnológicas de laminación o porque los estudios no han llegado a abarcar otras resistencias mayores, el acero que se emplea para las láminas metálicas para losas compuestas deberá tener una resistencia de fluencia nunca mayor a los $460 \mathrm{~N} / \mathrm{mm}^{2}$ (EU RO CO DE 4, 2004). 


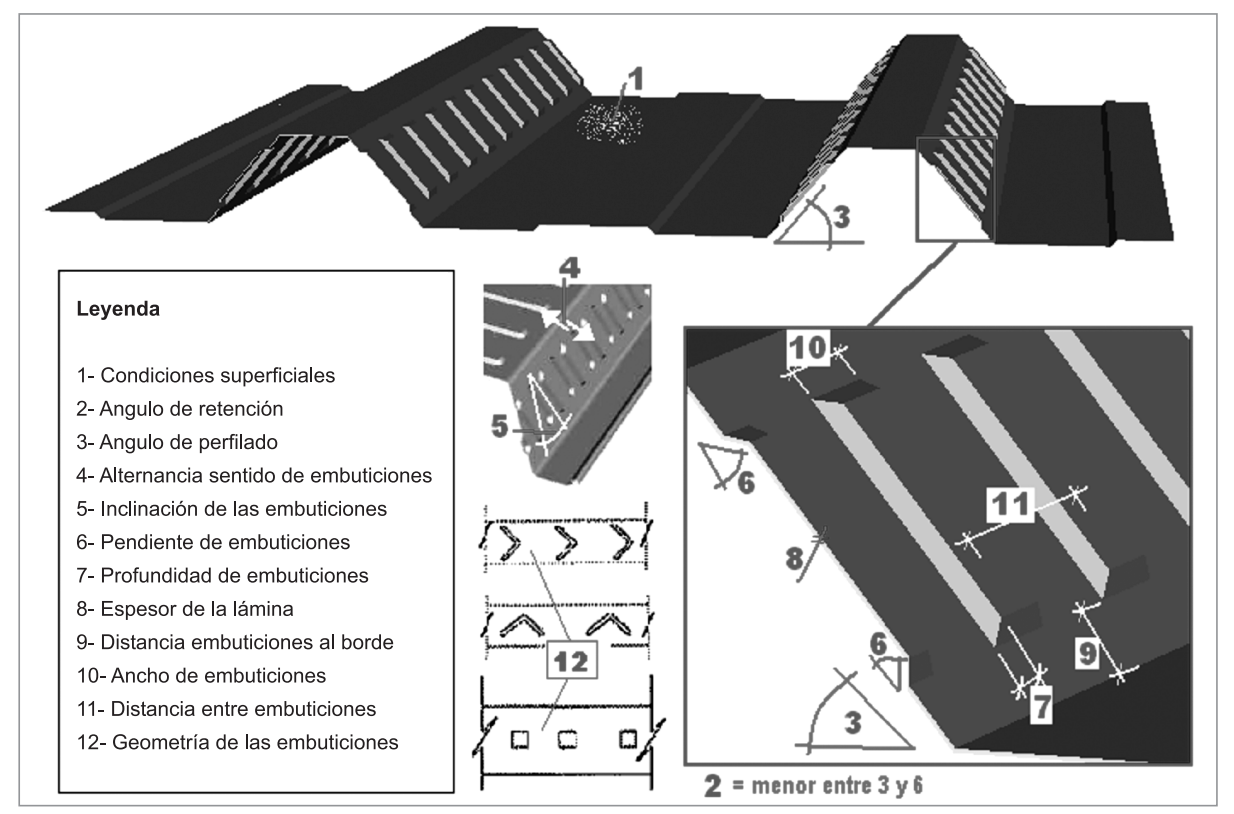

Figura 6. Parámetros que influencian comportamiento de las losas compuestas

\section{Condiciones iniciales para la creación de un nuevo steel deck}

Para proponer el diseño de una lámina cubana que sirva con fines colaborantes en las losas compuestas, o steel deck, se parte de emplear como base la tecnología existente en la Empresa de Estructuras M etálicas de Las Tunas, Cuba (METUNAS) y la teja grecada trapezoidal que en dicha fábrica se construye (Figura 7). (Tejas grecadas trapezoidales P4 250-40, en espesores desde $0.55 \mathrm{~mm}$ hasta $1 \mathrm{~mm}$ y longitudes entre 3500 y $12000 \mathrm{~mm}$ ).

La lámina tiene entre sus características más notables el de no contar con un peralto muy significativo, todo lo contrario, es una lámina bastante baja, lo cual reduce las ventajas estructurales de aprovechar la configuración geométrica para revertirla en trabajo a flexión como $T$ invertidas y aumentar la inercia del conjunto para retardar el fallo a flexión. Aún así se cuenta con la positiva experiencia de haber empleado satisfactoriamente esta lámina en su forma lisa como encofrado perdido (form deck) en algunas obras civiles, como la fábrica de cemento de Nuevitas, Camagüey, a partir de incorporar conectores de cortante en los extremos para tomar las fuerzas de igual nombre entre la lámina y el hormigón, sin que se halla presentado indicio alguno de tendencia a fallo por flexión. Un cambio de rodillos para peraltar un poco más la lámina traería como consecuencia un enorme costo de inversión, por lo que supusimos en primera instancia, a partir de la experiencia práctica, que el fallo más probable para esta lámina podría ser el debido al cortante longitudinal, tal como coinciden en apuntar los investigadores de distintas partes del mundo para la gran mayoría de los casos de losas compuestas (VainiÅnas et al., 2006).

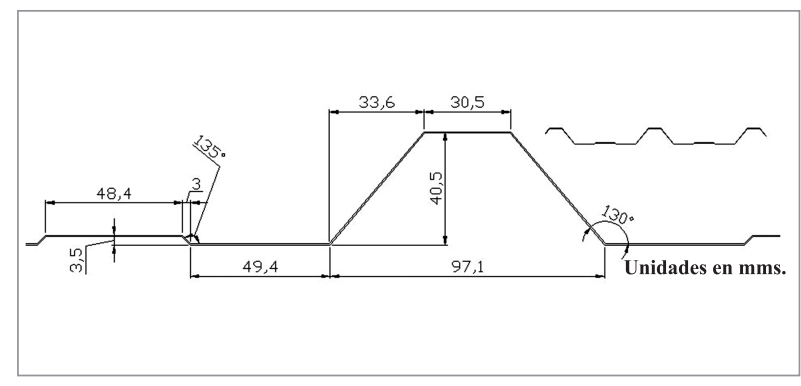

Figura 7. Geometría lámina grecada trapezoidal de METU NAS

Desde el punto de vista teórico chequeamos entonces, empleando el Método de Fluencia de Heagler (Heagler et al., 1992) para el caso que la interacción entre la lámina metálica y el bloque de hormigón fuera completa, en las condiciones geométricas más desfavorables, para qué luz máxima debería ocurrir el fallo a flexión. Las condiciones más desfavorables resultaron ser para el espesor más delgado de lámina $(0.55 \mathrm{~mm})$ en combinación con el mínimo espesor de carpeta de hormigón admitido por las normas, que es de $5 \mathrm{~cm}$; y para esas condiciones extremas los cálculos arrojaron que el fallo a flexión ocurriría para luces superiores a los 5 metros, lo cual es razón convincente que indica que el mejoramiento de esta lámina para que sirva con fines colaborantes en losas compuestas debe enfocarse en mejorar el sistema de retención mecánico para retardar el fallo por cortante longitudinal. 


\section{Método gráfico - analítico de optimización}

Para reducir la cantidad de ensayos a implementar, se propone un método gráfico- analítico para determinar el sistema de embuticiones óptimo. Este método tiene su base de implementación en los resultados experimentales de numerosos investigadores, y sobre todo en los de Ferrer (Ferrer, 2006), quien en su tesis de doctorado resumió las características y tendencias de varios de los parámetros que influencian el comportamiento de las losas compuestas. La implementación implica las láminas de perfilado abierto, que son las que más frecuentemente se construyen debido a las facilidades de fabricación y almacenaje que brinda su geometría.

Se caracterizaron cada uno de los parámetros que influencian el comportamiento estructural de las losas compuestas, y más específicamente su aporte con respecto a los esfuerzos de cortante longitudinal, y se determinó la función $V u=f($ parámetro) con su correspondiente coeficiente de correlación o confianza.

Este análisis se verificó para los siguientes parámetros y en los siguientes rangos (Figura 8): - ángulo de perfilado de la lámina metálica (AngPerf) [60ㅇ - 80으 (grados sexagesimales)

- espesor de la lámina metálica (LamEsp) [0.5mm $1.25 \mathrm{~mm}]$

- peralto de la lámina metálica (AltPerf) [35mm - 75mm]
- ancho de las grecas en la lámina metálica (AnG) [120mm - 150mm]

- rugosidad superficial (Rug) [0\% - 60\%]

- profundidad de las embuticiones (Eprof) [1mm - 3mm]]

- ancho de las embuticiones (Eanch) [7mm - 20mm]

- longitud de las embuticiones (Elong) [30mm - 55mm]

- pendiente de las paredes de las embuticiones (Epend) [350 - 50]

- distancia o paso entre embuticiones (Epaso) [35mm $70 \mathrm{~mm}$ ]

- inclinación de las embuticiones con respecto a la horizontal (Eincl) [50ㅇ - 70을

- resistencia del hormigón (HgonRes) [15N/mm² $\left.60 \mathrm{~N} / \mathrm{mm}^{2}\right]$

- espesor del bloque de hormigón (HgonEsp) [50mm $500 \mathrm{~mm}]$

El valor de cada uno de los parámetros participantes se puede modificar por separado. Esto es posible porque si se hace un análisis de estos se puede apreciar que todos son independientes, o sea, la alteración de uno no ejerce variación en ninguno de los restantes, siempre que se mantengan dentro del rango acotado o de dimensiones geométricas lógicas, por ejemplo: ancho de embutición debe ser menor o igual que el paso entre embuticiones.

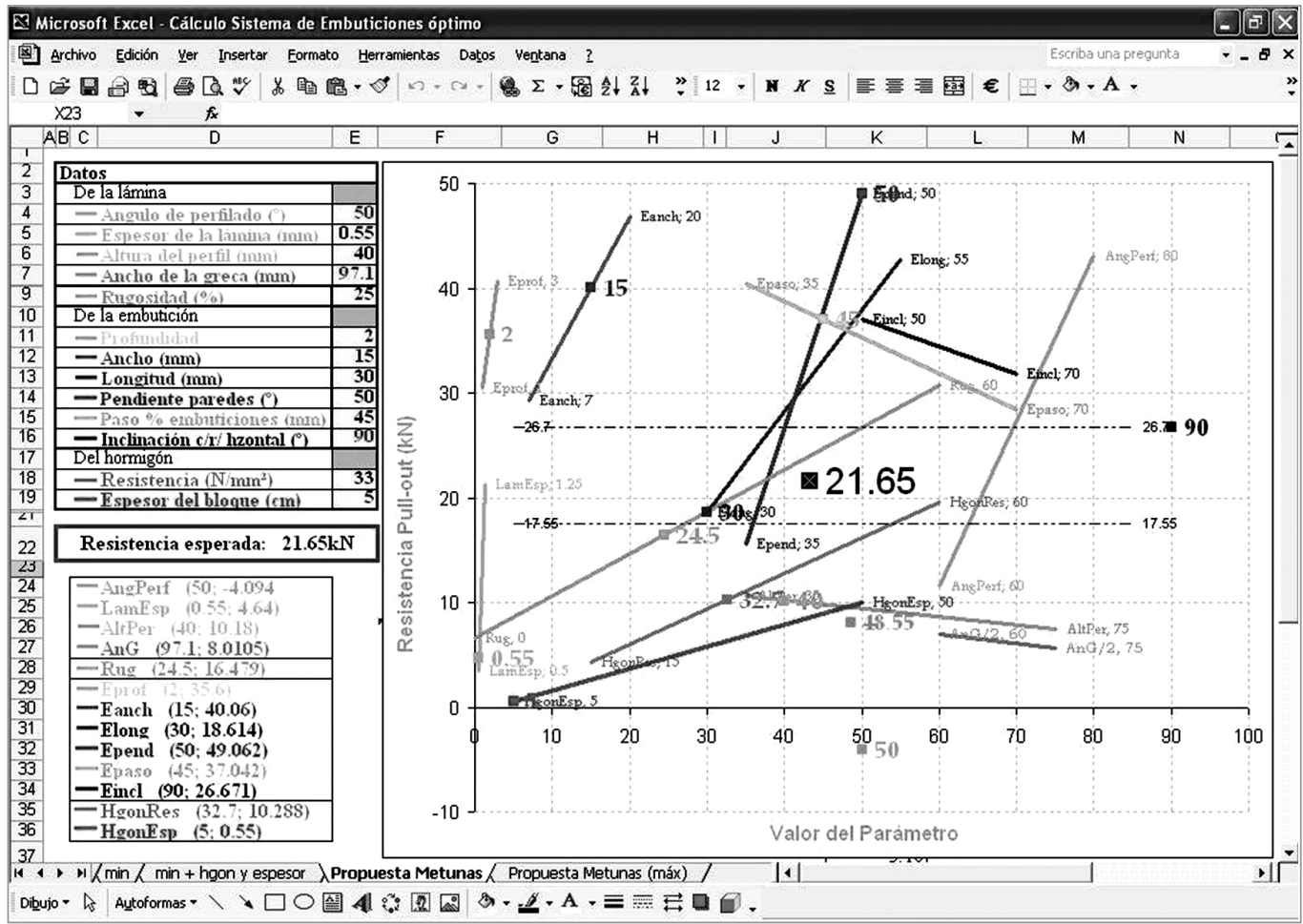

Figura 8. O ptimización de embuticiones para la lámina de METUNAS 
Para cada parámetro se ha determinado previamente con ensayos y/o modelaciones una tendencia, a partir de dejar todos los parámetros restantes fijos y variando solo el parámetro de estudio. Partiendo de esos resultados previos fue posible construir las ecuaciones de tendencia, y se pudo entonces valorar la magnitud de la influencia de cada parámetro en el resultado final. Luego, por análisis matemático fue posible determinar las funciones de peso para cada una de las variables y crear una función polinómica mixta que abarcara todos los parámetros analizados $V u=f($ parámetro 1, parámetro2,...parámetro13).

En la implementación (Figura 8) se puede observar la existencia de un valor central calculado, resaltado en negritas e indicado gráficamente con un punto (21.65). Por encima y por debajo de este valor se pueden apreciar dos líneas horizontales discontinuas también señalizadas con valores (17.55, 16.7). Éstas demarcan el rango de resistencias esperadas producto de la aleatoriedad del aporte de los parámetros participantes, y su separación con respecto al punto central está en función de los coeficientes de correlación que estos aportan.

Una limitante del método en cuanto a la exactitud de los valores de cálculo está dada en que si uno solo de los parámetros extralimita las cotas alcanzadas en los estudios previos a partir de los cuales se confeccionó el método, no se garantiza que la extrapolación sea totalmente consecuente con los resultados numéricos reales, por lo cual habrá entonces que acudir al sentido común del diseñador y no excederse demasiado fuera de los límites establecidos para evitar falsear los resultados, y que aún cuando el valor puntual no sea el más exacto, pueda servir a los fines de comparación y optimización del sistema de embuticiones, que es el objetivo principal de este método propuesto.

O bservando los gráficos podemos intuir que para una lámina de perfilado abierto, el máximo valor de resistencia a cortante se debe esperar para los puntos superiores extremos en el eje de las para cada una de las funciones evaluadas; sin embargo la teoría en escasas ocasiones acompaña a la práctica totalmente, y razones económicas y tecnológicas obligan a disminuir estos valores, por lo que una vez determinadas todas las condicionantes tecnológicas que existían en la empresa METUNAS, y siempre valorando la relación causa- efecto, prevaleciendo para este último la economía ante el reto de con un mínimo de inversiones lograr el producto más competitivo como fuera posible, fue necesario establecer nuevas cotas para los parámetros contribuyentes.

Con el empleo del software profesional ABAQUS 6.4 se modeló por Método de Elementos Finitos el proceso industrial de embutición en una lámina para comprobar que esta no sufriera daños ni desgarramientos, y verificar que las tensiones residuales propias del proceso de embutido no deformaran excesivamente la lámina (Figura 9).

El algoritmo utilizado en la modelación fue el de Newton- Raphson, que es un método iterativo para la solución de cualquier tipo de no linealidad. Esta elección fue necesaria porque en el fenómeno simulado aparecían grandes desplazamientos y cambios en la relación tensión-deformación del material debido a la notable plastificación del acero.

El modelo constitutivo utilizado para el acero hace empleo de las tensiones y deformaciones equivalentes de Von M ises para definir una ley uniaxial de fluencia multilineal con una ley simétrica de endurecimiento por deformación. Si la tensión equivalente de Von M ises, calculada bajo la hipótesis de deformaciones elásticas, supera el límite elástico inicial, entonces se inicia el cálculo de deformaciones plásticas.

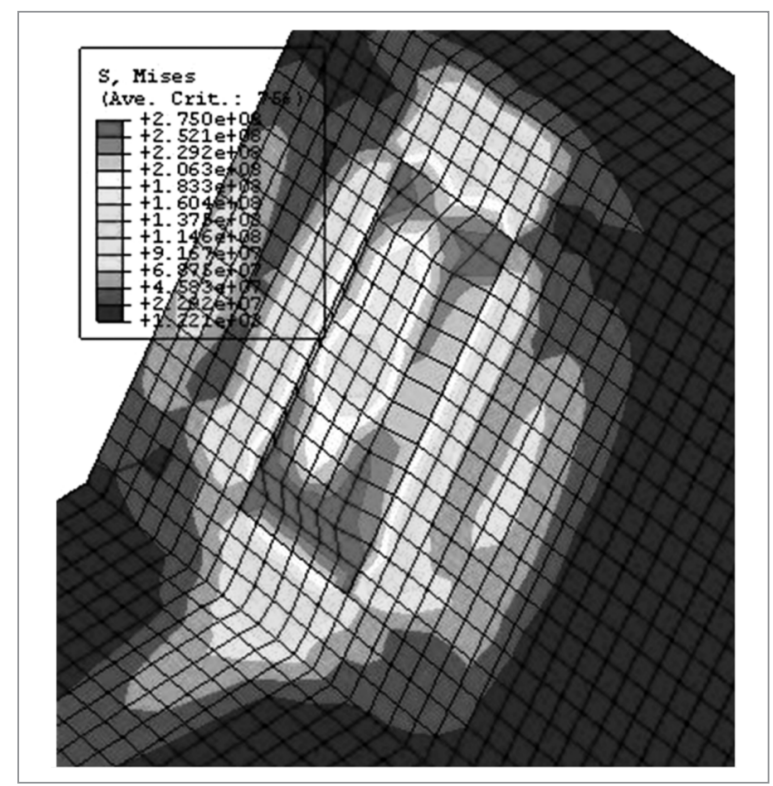

Figura 9. Modelación del proceso de embutición

Para el cálculo de las fuerzas normales de contacto (entre la lámina y los dispositivos de embutido), se empleó el método de penalización con multiplicador de Lagrange. La función de este multiplicador es incrementar las fuerzas de contacto elástico en caso de penetraciones excesivas, para mejorar así la convergencia. 
Para discretizar la lámina se escogió el elemento SHELL181, con el cual es posible el cálculo de deformaciones finitas además que considera los cambios de espesor causados por el propio proceso de deformación.

Se modeló para el espesor mínimo de lámina que es capaz de producir la línea de laminación de METU NAS, o sea: $0.55 \mathrm{~mm}$. La norma Europea recomienda que el espesor mínimo de las láminas colaborantes sea $0.7 \mathrm{~mm}$, no obstante realizamos la verificación para un espesor muy inferior para quedar completamente del lado de la seguridad.

Se simularon inicialmente penetraciones profundas $(4 \mathrm{~mm})$, pero estas desgarraban totalmente el material. Se fue entonces reduciendo progresivamente la profundidad de penetración hasta determinar que para $2.4 \mathrm{~mm}$ no existían los problemas temidos. De esta forma se decidió que la embutición debería tener como máximo $2 \mathrm{~mm}$ de profundidad (Aunque para $0.7 \mathrm{~mm}$ de espesor, una profundidad de embutición de $2.5 \mathrm{~mm}$ no acusa problemas de ninguna índole).

Se ratificó la veracidad de los resultados obtenidos en la modelación empleando un troquel rústico y una prensa, los cuales fueron suficientes para crear embuticiones en láminas lisas, y por inspección visual y con ayuda de un medidor de espesor se ratificó que, tal y como se predijo en la modelación, para profundidad de embutición de $2 \mathrm{~mm}$ en láminas de espesor $0.55 \mathrm{~mm}$ no aparecían signos de desgarramiento, rotura 0 deformación inadmisible en el material. Posteriormente, dispositivos con geometría similar fueron emplazados en un troquel para, una vez instalado en una prensa hidráulica, poder practicar las embuticiones en una lámina con la configuración grecada real (Figura 10).

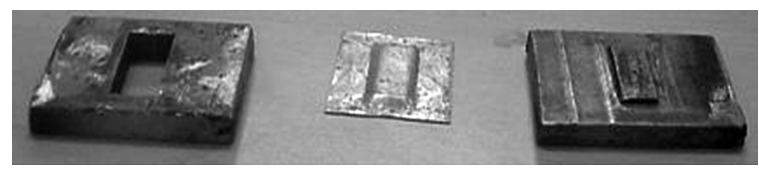

Figura 10. Medios rústicos para comprobar embutido en lámina plana

El paso entre embuticiones se determinó a partir de la modelación de Elementos Finitos bajo el criterio de que la zona de influencia de tensiones generadas por la fabricación por punzonamiento de una embutición, no se solapara con la de la embutición vecina.

La fabricación semi- artesanal de las embuticiones en tramos de lámina grecada (Figura 11) sirvió además para corroborar otras dos predicciones del modelo numérico, la primera, que en embuticiones de esquinas rectas, dichas esquinas son zonas de altas concentraciones de tensiones (Figura 9), por lo que se recomienda redondearlas ligeramente. La segunda observación es que el mapa de tensiones generadas durante el proceso de embutido indica que estas son más intensas y se extienden en una longitud mayor, en las zonas adyacentes al ancho de las embuticiones (Figura 9), por tanto, si la distancia de las embuticiones al borde es insuficiente, los bordes de las láminas pueden sufrir deformaciones apreciables.

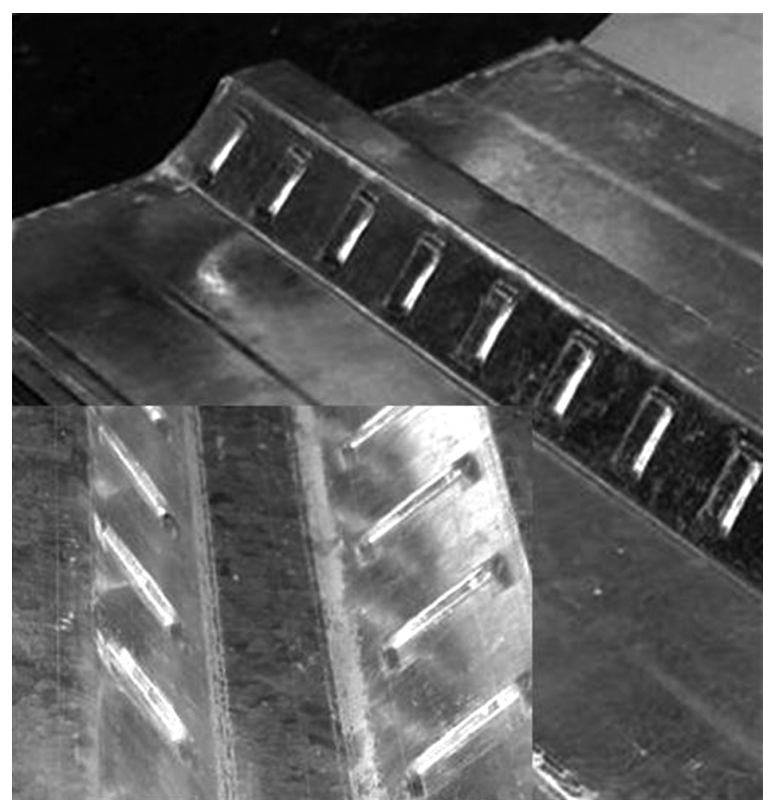

Figura 11. Embuticiones en lámina M ETUNAS

Así se concluye que teórica y tecnológicamente posible, el mejor sistema de embuticiones a introducir en la lámina de METU NAS deberá tener las siguientes características geométricas:

Profundidad de la embutición: $2 \mathrm{~mm}$

Ancho de la embutición: $15 \mathrm{~mm}$ Longitud de la embutición: $30 \mathrm{~mm}$ Pendiente de las paredes verticales: 50 은 Paso entre embuticiones: $45 \mathrm{~mm}$ Inclinación de la embutición con respecto a la horizontal: 90 응

Q ue en una losa compuesta con carpeta de $5 \mathrm{~cm}$ de espesor de losa, con resistencia del hormigón $17.5 \mathrm{~N} / \mathrm{mm}^{2}$, rugosidad superficial del $25 \%$ y los espesores de lámina a continuación indicados se esperaría una resistencia a cortante de (ver Tabla 1 ): 
Tabla 1. Espesores de láminas

\begin{tabular}{||c|c|c|c||}
\hline \multirow{2}{*}{ Espesor lámina } & \multicolumn{3}{|c|}{ Resistencia a Cortante Esperada (KN) } \\
\cline { 2 - 4 } & Valor Central & Cota mínima & Cota máxima \\
\hline 0.7 & 19.27 & 15.62 & 23.77 \\
\hline 0.8 & 21.65 & 17.55 & 26.70 \\
\hline 1.0 & 27.60 & 22.38 & 34.04 \\
\hline
\end{tabular}

Así queda propuesto un método de optimización a través del cual resulta posible estimar el sistema de embuticionesóptimo a introducir en una lámina de perfilado abierto, y determinar con cierto grado de exactitud la resistencia a cortante esperada, de forma totalmente analítica, sin necesidad de los costosos ensayos de flexión a escala natural, pues los datos necesarios para la estimación son puramente geométricosy de resistencia de los materiales participantes.

\section{Conclusiones}

- El fallo más probable de ocurrir en las losas compuestas con lámina metálica colaborante es el debido al cortante longitudinal. Para aplazar la ocurrencia de este tipo de fallo se persigue aumentar la interacción mecánica entre el hormigón y el acero a través de embuticiones en la lámina.

- Debido a la no linealidad de los fenómenos de interacción y a la gran diversidad de tipologías de láminas, los investigadores no han podido establecer un método de cálculo totalmente analítico para caracterizar este tipo de elementos estructurales, sino que se ha tenido que recurrir a métodos empíricos.

- El método experimental más incuestionable es el que reproduce a escala natural el fenómeno, que es el más fiable, pero también el más costoso. La ecuación que respondiendo a este diseño de experimento más se adecua al carácter físico real es la propuesta por el Eurocódigo: el Método de Interacción Parcial de Cortante (PSC), y entre las ecuaciones para determinar los parámetros m-k, la más fiable es la propuesta por Seleim 1979.

- Empleando el método de interacción parcial de cortante se logran diseños más económicos que si se emplean cualquiera de los métodos m-k, sin embargo este método no es fiable cuando se espera la ocurrencia de fallos frágiles.

- Las losas compuestas de tramos continuos son más resistentes entre un 10 y un $15 \%$ que las simplemente apoyadas que se prueban a flexión simple en el ensayo a escala natural.

- El desarrollo de ensayos a escala reducida intenta reproducir con mayor o menor fidelidad el fenómeno de deslizamiento longitudinal. Uno de los más aceptados universalmente es el de Daniels 1988, para quien se creó en el 2004 un método que los autores
Ilamaron Nuevo Método Simplificado.

- Los ensayos a escala reducida son más fáciles de reproducir por método de elementos finitos que los de escala natural, pues estos últimos generarían debido a una discretización medianamente refinada millones de ecuaciones que serían imposibles de resolver empleando ordenadores convencionales.

- El método de elementos finitos se puede emplear satisfactoriamente para determinar las tensiones residuales propias de los procesos de laminación y embutido durante el proceso industrial de fabricación de las láminas colaborantes.

- Se pueden distinguir dos grandes grupos de láminas metálicas: de perfil reentrante y de perfil con nervios abiertos. Estos últimos, aunque son los menos eficientes estructuralmente, son los más fáciles de reproducir a escala industrial y más fáciles de manipular y almacenar.

- Analizando ensayos practicados por otros investigadores a distintas láminas de nervios abiertos, se puede determinar la influencia de los diferentes parámetros que influencian el comportamiento estructural de las losas compuestas. O ptimizando cada uno de los parámetros participantes en el diseño, se perfeccionaría en su conjunto el comportamiento estructural de las losas compuestas.

- Empleando métodos numéricos se puede determinar un estimado de resistencia a cortante para una losa con lámina de nervios abiertos, ahorrando así para los productores de lámina la necesidad de ensayos de tanteo para determinar el sistema de embuticiones óptimo a practicar en un nuevo producto.

- Con un coste reducido de inversiones y aprovechando la tecnología disponible en METUNAS sería posible producir en Cuba a escala industrial una lámina provista de embuticiones que sirva con efectos colaborantes para las losas compuestas.

\section{Referencias}

Abdullah R. (2004), Experimental Evaluation And Analytical M odeling O f Shear Bond In Composite Slabs.

Abdullah R. y Easterling W. S. (2007), Determination Of Composite Slab Strength Using A New Elemental Test M ethod. Joumal Of Structural Engineering, 133.

AISC (2007) AISC, Steel D esign After College.

ASTM-E8-00B (2001), ASTM E8-00b Standard Test M ethods For Tension Testing Of M etallic Materials.

Bode H. y Dauwel T. (1999), Steel-Concrete Composite Slabs. Design Based On Partial Connection. Steel And Composite Structures Intemational. Deft, Holanda.

Bode H. y Sauerborn I. (1992), M odern Design Concept For Composite Slabs With Ductile Behaviour. IN ASCE (Ed.) Engineering Foundation Conference On Composite Construction In Steel And Concrete II.

Chen S. (2003), Load Carrying Capacity Of Composite Slabs W ith 
Various End Constraints. Joumal Of Constructional Steel Research, 59, 385-403.

Crisinel M. y Edder P. (2004), New Method For The Design Of Composite Slabs Composite Construction In Steel And Concrete V.

Crisinel M., Ferrer Ballester M. y Rossich M. (2006), Influence Of Sheet Surface Conditions And Concrete Strength On The Longitudinal Shear Resistance of Composite Slabs. Crisinel M., Guignard P. y Schumacher A. (1999), Steel-Concrete Composite Slabs. Progress Report ECCA TWG 7.6. Steel And Composite Structures International Deft, Holanda. CSSBI-S3 (2003), CSSBI Criteria For The Design Of Composite Slabs. Daniels B. (1988), Shear Bond Pull-O utTests For Cold-Formed-Steel Composite Slabs. IN PU BLICACIÓ N, I. (Ed.) Lausanna, Escuela Politécnica Federal De Lausanna.

Daniels B. y Crisinel M. (1993), Composite Slab Behavior And Strength Analysis. Part II: Comparisons W ith Test Results And Parametric Analysis. Joumal Of Structural Engineering, 119, 3649.

Edder P., Crisinel M. (2003), Nouvelle Approche Pour Le Dimensionnement Des Dalles Mixtes. IN ICOM (Ed.) ICOM 472. Lausanne, Laboratoire De La Construction Métallique (ICOM). Eurocode-4 (2004), Eurocode 4: Design Of Composite Steel And Concrete Structures: General Rules And Rules For Buildings. Ferrer M. (2006), Estudio N umérico Y Experimental De La Interacción Entre La Chapa DeAcero Y $\mathrm{E}$ Homigón Para La M ejora Resistente De Las Losas. Barcelona, U niversidad Politécnica De Cataluña (UPC).

Ferrer M., Marimóm F. y Crisinel M. (2007), Designing Cold-Formed Steel Sheets For Composite Slabs: An Experimentally Validated FEM Approach To Slip Failure M echanics. Elsevier Ltd, 44, 11.

Ferrer M., Marimon F., y Roure F. (2005), Design M ethodology Of Profiled Steel Sheets For Composite Slabs By FEM. European Action COST C12: Improving Buildings' Structural Q uality By New Technologies.

Heagler R. B., Luttrell L. D. y Easterling W. S. (1992), The Steel Deck Institute Method For Composite Slab Design. IN ASCE (Ed.) Engineering Foundation Conference O n Composite Construction In Steel And Concrete II.

Hemández D. L. (2003), Cálculo De Estructuras Compuestas De Hormigón Y Acero A Flexión Con El Empleo De Láminas Perfiladas.

Jolly C. y Zubair A. (1987), Efficiency Of Shear-Bond Interlock Between Profiled Steel Sheeting And Concrete. Composite Steel Structures. Advances, Design, And Construction, 127-136.

Kitoh H. y Sonoda K. (1996), Bond Characteristics Of Embossed Steel Elements. IN ASCE (Ed.) Engineering Foundation Conference On Composite Construction In Steel And Concrete III.

Laane A. y Edder P. (2002), Pull-O ut Tests On Steel-Concrete Composite Slab Small-Scale Specimen. IN ICOM (Ed.) ICOM 451. Lausanne, Laboratoire De La Construction Métallique (ICOM).

Luttrell L. D. (1987), Flexural Strength Of Composite Slabs. Composite Steel Structures. Advances, Design, And Construction, 106-116.

Makelainen P. y Sun Y. (1999), The Longitudinal Shear Behaviour Of A New Steel Sheeting Profile For Composite Floor Slabs. Journal Of Constructional Steel Research, 49, 117-128.
M arimuthu V., Seetharaman S., A rul Jayachandran S., Chellappan A., Bandyopadhyay, T. K. y D utta D. (2006), Experimental Studies On Composite Deck Slabs To Determine The Shear Bond Characteristic (M-K) Values Of The Embossed Profiled Sheet. Journal Of Constructional Steel Research, 63, 791-803.

N C-082 (2004), NC 082:2004 Cálculo De Losas Compuestas Con Láminas De Acero Como Encofrado Colaborante Sometidas A Cargas Estáticas. Código De Buenas Prácticas. IN N C (Ed.) 1 Ed., MICONS.

Porter, M. y Ekberg, C. (1975), Design Recommendations For Steel Deck Floor Slabs. Third Intermational Specialty Conference On Cold-Formed Steel Structures. Universidad De M issouri-Rolla.

Porter M. y Ekberg C. (1976), D esign Recommendations For Steel Deck Floor Slabs. ASCE Joumal OfThe Structural Division, 102, 2121-2136.

Ranzi G., Ansourian P., Gara F., Leoni G. y Dezi L. (2005), Displacement Based Formulation Of Composite Beams With Longitudinal Slip And Vertical U plift.

Rossich M. (2005), Analyse Et Développement De L'Essai De Cisaillement Pull O ut. ICOM. Laussanna, Escuela Politécnica Federal De Laussanna.

Schumacher A., Laane, A. y Crisinel M. (2000), Development Of A New Design Approach For Composite Slabs. IN ASCE (Ed.) Engineering Foundation Conference On Composite Construction In Steel And Concrete IV.

Schuster R. M . (1970), Strength And Behavior OfCold-Rolled SteelDeck Reinforced Concrete Floor Slabs. Ames, lowa, U niversidad De lowa.

Schuurman R. G., y Stark J. W. B. (1996), Longitudinal Shear Resistance Of Composite Slabs. To A Better Understanding Of Physical Behaviour. IN ASCE (Ed.) Engineering Foundation Conference O n Composite Construction In Steel And Concrete III.

Seleim S. (1979), Ultimate Shear-Bond Capacity Of Composite Steel Deck Concrete Slabs. Waterloo, O ntario, U niversidad De Waterloo.

Seleim S., y Schuster R. M. (1985), Shear-Bond Resistance Of Composite Deck-Slabs. Canadian Journal Of Civil Engineering, 12, 316-324.

Tenhovuori A. I. y Leskela M . V. (1998), Longitudinal Shear Resistance Of Composite Slabs. Journal Of Constructional Steel Research, $46,1-3$.

Vainiånas P., Valivonis J., M aräiukaitis G. y Jonaitis B. (2006), Analysis Of Longitudinal Shear Behaviour For Composite Steel And Concrete Slabs. Journal Of Constructional Steel Research, 62, 1264-1269.

Veljkovic M. (1994), Sheeting-Concrete Interaction Performances In The Composite Floor Slab. Nordic Concrete Research, 18.

Veljkovic M . (2000), Behaviour And Design Of Shallow Composite Slab. IN ASCE (Ed.) Engineering Foundation Conference O n Composite Construction In Steel And Concrete IV. Veljkovic M. y Johansson B. (2001), Partial Interaction In Composite Slabs (Final Report Of BFR Project). Division Of Steel Structures, 2001:03.

Virdi K. S. (2006), Finite Difference Method For Nonlinear Analysis Of Structures. Joumal Of Constructional Steel Research, 62, 12101218. 